\title{
An altered posterior question-mark incision is associated with a reduced infection rate of cranioplasty after decompressive hemicraniectomy
}

\author{
Michael Veldeman, MD, ${ }^{1}$ Lorina Daleiden, MD, ${ }^{1,2}$ Hussam Hamou, MD, ${ }^{1}$ Anke Höllig, MD, ${ }^{1}$ and \\ Hans Clusmann, MD' \\ 'Department of Neurosurgery, RWTH Aachen University Hospital, Aachen; and 2Department of Neurosurgery, Military Hospital \\ Koblenz, Germany
}

\begin{abstract}
OBJECTIVE Performing a cranioplasty (CP) after decompressive craniotomy is a straightforward neurosurgical procedure, but it remains associated with a high complication rate. Surgical site infection (SSI), aseptic bone resorption (aBR), and need for a secondary CP are the most common complications. This observational study aimed to identify modifiable risk factors to prevent $\mathrm{CP}$ failure.
\end{abstract}

METHODS A retrospective analysis was performed of all patients who underwent $\mathrm{CP}$ following decompressive hemicraniectomy (DHC) between 2010 and 2018 at a single institution. Predictors of SSI, aBR, and need for allograft CP were evaluated in a univariate analysis and multivariate logistic regression model.

RESULTS One hundred eighty-six patients treated with $\mathrm{CP}$ after $\mathrm{DHC}$ were included. The diagnoses leading to a DHC were as follows: stroke (83 patients, $44.6 \%$ ), traumatic brain injury (55 patients, $29.6 \%$ ), subarachnoid hemorrhage (33 patients, 17.7\%), and intracerebral hemorrhage (15 patients, 8.1\%). Post-CP SSI occurred in 25 patients (13.4\%), whereas aBR occurred in 32 cases (17.2\%). An altered posterior question-mark incision, ending behind the ear, was associated with a significantly lower infection rate and $\mathrm{CP}$ failure, compared to the classic question-mark incision $(6.3 \%$ vs $18.4 \% ; p=0.021$ ). The only significant predictor of aBR was patient age, in which those developing resorption were on average 16 years younger than those without aBR $(p<0.001)$.

CONCLUSIONS The primary goal of this retrospective cohort analysis was to identify adjustable risk factors to prevent post-CP complications. In this analysis, a posterior question-mark incision proved beneficial regarding infection and CP failure. The authors believe that these findings are caused by the better vascularized skin flap due to preservation of the superficial temporal artery and partial preservation of the occipital artery. In this trial, the posterior question-mark incision was identified as an easily and costless adaptable technique to reduce $\mathrm{CP}$ failure rates.

https://thejns.org/doi/abs/10.3171/2020.2.JNS193335

KEYWORDS cranioplasty; decompressive hemicraniectomy; complications; wound infection; surgical site infection; aseptic bone resorption; osteonecrosis; surgical technique

$\mathrm{D}$ ECOMPRESSIVE hemicraniectomy (DHC) is a routine neurosurgical procedure that can be lifesaving in cases of refractory increased intracranial pressure. Apart from classic indications for its use such as in severe traumatic brain injury (TBI) ${ }^{1}$ and malignant cerebral ischemia, ${ }^{2-4}$ the procedure has become more common in aneurysmal subarachnoid hemorrhage (SAH), 5,6 and its effectiveness in intracerebral hemorrhage (ICH) is currently being investigated in a randomized controlled trial., ${ }^{7,8}$ The conditions requiring this sort of treatment are severe and often cause considerable neurological impairment. As indications for the procedure broaden, more patients will

ABBREVIATIONS aBR = aseptic bone resorption; $\mathrm{CP}=$ cranioplasty; $\mathrm{CRP}=\mathrm{C}$-reactive protein; $\mathrm{DHC}=$ decompressive hemicraniectomy; $\mathrm{DM}=$ diabetes mellitus; $\mathrm{EVD}=$ external ventricular drain; GOS-E = Glasgow Outcome Scale-Extended; ICH = intracerebral hemorrhage; ICU = intensive care unit; MMI = malignant middle cerebral artery infarction; PMMA = polymethyImethacrylate; $\mathrm{SAH}=$ subarachnoid hemorrhage; $\mathrm{SSI}$ = surgical site infection; STA = superficial temporal artery; TBI = traumatic brain injury; WBC = white blood cell.

SUBMITTED December 10, 2019. ACCEPTED February 4, 2020.

INCLUDE WHEN CITING Published online April 24, 2020; DOI: 10.3171/2020.2.JNS193335. 
require cranial reconstructive surgery. After stabilization of the underlying condition and once the swelling has subsided, a cranioplasty (CP) is required to reconstruct the skull defect. This is to offer cerebral protection and cosmetic repair but also to restore CSF dynamics and normal pressure conditions within the cranial vault. $\mathrm{CP}$ may also improve neurocognitive functioning, and early $\mathrm{CP}$ can even enhance this effect. ${ }^{9}$ Although $\mathrm{CP}$ is a straightforward procedure, the complication rate remains rather high. Complications included surgical site infection (SSI) with or without wound dehiscence, aseptic bone resorption (aBR), postoperative bleeds, epidural fluid collections or hygroma, seizures, and late hydrocephalus. ${ }^{10}$ These complications can lead to severe additional morbidity and even death. Depending on what is considered a complication of the $\mathrm{CP}$ itself, complication rates in the literature range between $12.3 \%$ and $29.7 \% .{ }^{11-14}$ Most complications trigger additional surgery consisting of bone flap removal, antibiotic treatment if necessary, and eventually the need for a secondary CP.

In recent years a multitude of studies have been published addressing the problem of complications after CP. Many trials have focused on patient-specific risk factors such as obesity, diabetes mellitus (DM), smoking, arterial disease, hypertension, alcohol abuse, and drug abuse..$^{14,15}$ Although these are typical risk factors for SSI in general surgery or spine procedures, they do not play a major role in CP failure. Artificial allograft implants have been introduced that are made out of materials such as titanium, polyetheretherketone, polymethylmethacrylate (PMMA), and hydroxyapatite without any relevant effect on infection rates..$^{16-18}$ The cryopreserved $\left(\right.$ at $-80^{\circ} \mathrm{C}$ ) autologous bone flap remains the gold standard for primary cranial repair as it is cheap, readily available, and has an ideal geometric fit. Longer operative times and temporalis muscle resection have been identified as modifiable risk factors of SSI ${ }^{19-21}$ Blind antibiotic topical application during $\mathrm{CP}$ failed to reduce post-CP infection rates. ${ }^{22}$

The focus of this analysis is on identifying modifiable patient- or surgery-specific risk factors contributing to $\mathrm{CP}$ failure.

\section{Methods}

\section{Patient Population and Study Design}

We retrospectively analyzed all patients treated by DHC in a single institution (University Hospital Aachen, Germany) between 2010 and 2018. Data were obtained from hospital records, extracting all relevant variables and documented complications. SSI was defined as an open or closed graft infection requiring surgical wound revision with or without the removal of the implanted material. This was due to either a visible wound infection or a positive infectious lumbar puncture. Aseptic superficial wound healing problems not requiring surgical intervention or antibiotic treatment were not included. aBR was defined as significant thinning of the bone flap on CT scan, palpable soft spot, or resorption leading to an unsatisfactory cosmetic result. If brain protection was compromised due to aBR, an allograft revision $\mathrm{CP}$ was considered. This study was registered as ISRCTN46696883 in the ISRCTN registry (http://www.isrctn.com/ISRCTN46696883). This paper is written in accordance with the STROBE statement for reporting observational studies in epidemiology. The trial was conducted in accordance with the recommendations of the ethics committee of the Medical Faculty of the RWTH Aachen University.

The inclusion criteria were prefixed and defined as all DHC patients who were treated between 2010 and 2018 by autologous $\mathrm{CP}$, between 16 and 90 years of age, for malignant middle cerebral artery infarction (MMI), TBI, $\mathrm{SAH}$, or ICH. Clinical follow-up had to exceed 12 months after $\mathrm{CP}$ and had to include at least a clinical neurological examination with inspection of the wound and bone flap with or without CT. Patients were excluded when the DHC was performed for other reasons than named above, such as for infection (interference with infection rate) and after tumor surgery (usually secondary DHC). Bifrontal decompressive craniectomies, children younger than 16 years (before completion of skull growth), immunocompromised patients (iatrogenic or acquired), or patients with a follow-up of less than 12 months were also excluded.

\section{Standard Treatment Algorithm}

Prior to every procedure, patients received a single shot of third-generation cephalosporin (cefuroxime) during anesthesia induction, or in the case of allergy/cross-allergy, clindamycin was used. A unilateral frontotemporoparietal DHC was performed in a rapid closure technique without duraplasty. ${ }^{23,24}$ The anteroposterior diameter of the bone flap was always kept above $12 \mathrm{~cm} .{ }^{25}$ As first choice, independent of age, all patients received their cryopreserved $\left(-80^{\circ} \mathrm{C}\right)$ autologous bone flap. The time point of $\mathrm{CP}$ was not fixed and depended on the development of the rehabilitation process. Ultra-early ( $<30$ days) $\mathrm{CP}$ was avoided if possible due to an observed higher rate of infections and CSF fistulas and hydrocephalus. ${ }^{26}$ Prior to reinsertion, the autologous bone flap was defrosted, the skin incision was reopened, and the bony rims of the skull defect were prepared. The pseudodural plane was meticulously dissected to prevent CSF leaks. Additional attention was paid to preservation of superficial temporal artery (STA) branches. The bone flap was fixed with at least 6 nonabsorbable polyethylene sutures. A minimum of 5 central sutures were applied to fixate the dural layer to the bone flap. Two nonsuction drains were left behind in the subgaleal space during closure.

\section{Incision Types}

Three different incision types for the initial DHC were routinely used during the study inclusion period. The first consists of the standard large reversed question-mark incision starting $1 \mathrm{~cm}$ anterior to the tragus at the temporal root of the zygoma, curving posteriorly above and behind the ear extending to the midline at the inion, and then turning anteriorly to end at the widow's peak. During opening, a primary preservation of the STA was always attempted via careful dissection. Accidental scarification occasionally occurred in the case of a very posteriorly located main arterial stem, or partially in the case of a low branching-off of the parietooccipital portion of the STA. 
An altered posterior question-mark skin incision was used starting behind the ear, slightly posterior to the base of the mastoid process. The incision is carried cranially across the lambdoid and along the sagittal suture to reach the widow's peak. ${ }^{27}$

A third type of incision was implemented out of necessity, in cases in which a pterional craniotomy had been performed beforehand. This "n-type" incision is mostly used in patients with SAH previously treated by operative clipping. ${ }^{28}$ The incision starts perpendicular to and at the middle of the previous frontotemporal incision and runs posteriorly to reach the inion, after which it curves laterally in the direction of the mastoid base. The "T-type" or "Kempe" incision was abandoned before 2010 due to frequent wound healing problems at the intersection of both linear incisions. In hydrocephalic patients, shunting was performed before or after, not during, $\mathrm{CP}$ due to documented reduced infection rates with timely separated procedures. ${ }^{29-31}$ The different incision types are depicted in Fig. 1. The diagnosis of relevant aBR or SSI resulted in bone flap removal, and in the case of the latter, antibiotic treatment for 2-4 weeks. Allograft PMMA computeraided personalized implants were used as the secondary $\mathrm{CP}$ material. Our institutional treatment algorithm is illustrated in Fig. 2.

\section{Risk Factors}

Patient-specific risk factors were extracted from hospital records, such as prevalence of obesity (BMI > 30), DM, smoking, arterial disease (coronary or peripheral), hypertension, alcohol and drug abuse, length of initial intensive care unit (ICU) stay, as well as pre-CP white blood cell (WBC) count and C-reactive protein (CRP) levels (suggestive of concomitant underlying infections). In addition, the clinical neurological state defined by the extended Glasgow Outcome Scale (GOS-E) at the time of CP and whether the patient was bedridden or not was noted. Surgery-specific variables were registered, such as the time between DHC and CP dichotomized into early ( $<90$ days) and late (> 90 days) $\mathrm{CP}$, the duration of $\mathrm{CP}$ surgery, and the incision type. The occurrence of SSI after DHC, the presence of an external ventricular drain (EVD) during the initial hospital stay, and the need for permanent shunt placement was also noted.

\section{Outcome Parameters}

The primary outcome was defined as the occurrence of SSI triggering surgical revision. Additional secondary outcome parameters were the occurrence of aBR and the need for allograft CP.

\section{Statistical Analysis}

All data are presented as the mean and standard deviation for normally distributed variables. Categorical variables are depicted as frequencies and proportions. A univariate analysis was applied to screen for independent variables affecting the occurrence of SSI, aBR, and the need for allograft CP. After normality testing via plotting and the Shapiro-Wilk test, the appropriate statistical test was selected. For nominal data, the chi-square test was used, for normally distributed continuous data the unpaired t-test was used, and for nonnormally distributed data the Mann-Whitney U-test was used. Missing outcome data were not imputed. Predictive factors were further analyzed in a multivariate logistic regression model. All statistical analyses were performed using IBM SPSS (version 25, IBM Corp.). Statistical significance was defined as a two-sided $\mathrm{p}$ value $<0.05$.

\section{Results \\ Patients}

Between 2010 and 2018, a total of 387 patients were treated by DHC in our department. Of those, 129 died before the CP could be performed. An additional 60 patients were lost to follow-up. Two patients were excluded because the DHC was performed for meningitis-caused generalized edema. Primary autologous CP was performed in 196 patients, and 10 additional patients in this group were lost to follow-up. This resulted in 186 patients treated with $\mathrm{CP}$ after DHC with a minimum of 12 months of clinical follow-up. The indications for DHC were MMI (83 patients, 44.6\%), TBI (55 patients, 29.6\%), SAH (33 patients, 17.7\%), and ICH (15 patients, $8.1 \%)$. The study enrollment process is depicted in Fig. 3. Baseline characteristics of the cohort are summarized in Table 1 . The mean patient age was 49 \pm 16 years, with a female/male ratio of 77:109. The mean duration of follow-up was $1153 \pm 703$ days.

\section{Complications}

Post-CP SSI occurred in 25 patients (13.4\%). aBR occurred in 32 cases $(17.2 \%)$. Bone resorption triggered secondary $\mathrm{CP}$ in 12 patients (6.4\%). In 2 cases, the autologous bone flap needed to be removed shortly after surgery due to unanticipated post-CP brain swelling. This led to a total of 43 patients $(23.1 \%)$ requiring secondary allograft CP. In 2 SSI cases more than one causal pathogen was identified. Staphylococcus aureus was the most often encountered pathogen, in a total of 9 cases. Other common species in descending order of prevalence were S. epidermidis in 4 cases, Escherichia coli in 3 cases, and Enterococcus faecalis, Pseudomonas spp., and Proteus mirabilis each in 1 case. In 2 cases, multidrug-resistant bacteria were identified (methicillin-resistant $S$. aureus [MRSA] and multidrug resistant gram-negative bacteria). Cutibacterium acnes was identified as a common contaminant. Bone resorption triggered flap removal and direct allograft $\mathrm{CP}$ in 12 patients (6.4\%), 8 of whom were younger than 30 years of age.

\section{Primary Outcome}

In a univariate analysis, significant predictors for SSI were patient age $(p=0.041)$, early $\mathrm{CP}(\mathrm{p}=0.025)$, and incision type $(p=0.034)$. No significant differences in age were observed between the SSI subgroup (mean age $46 \pm$ 15 years) versus the no-SSI subgroup (mean age $50 \pm 16$ years, $\mathrm{p}=0.226$ ). Ten patients in the early-CP subgroup developed SSI (16.4\%) versus 15 (12.0\%) in the late-CP group $(\mathrm{p}=0.493)$. In a multivariate regression analysis, only the incision type was associated significantly with the rate of SSI. The question-mark incision group $(\mathrm{n}=$ 
Veldeman et al.
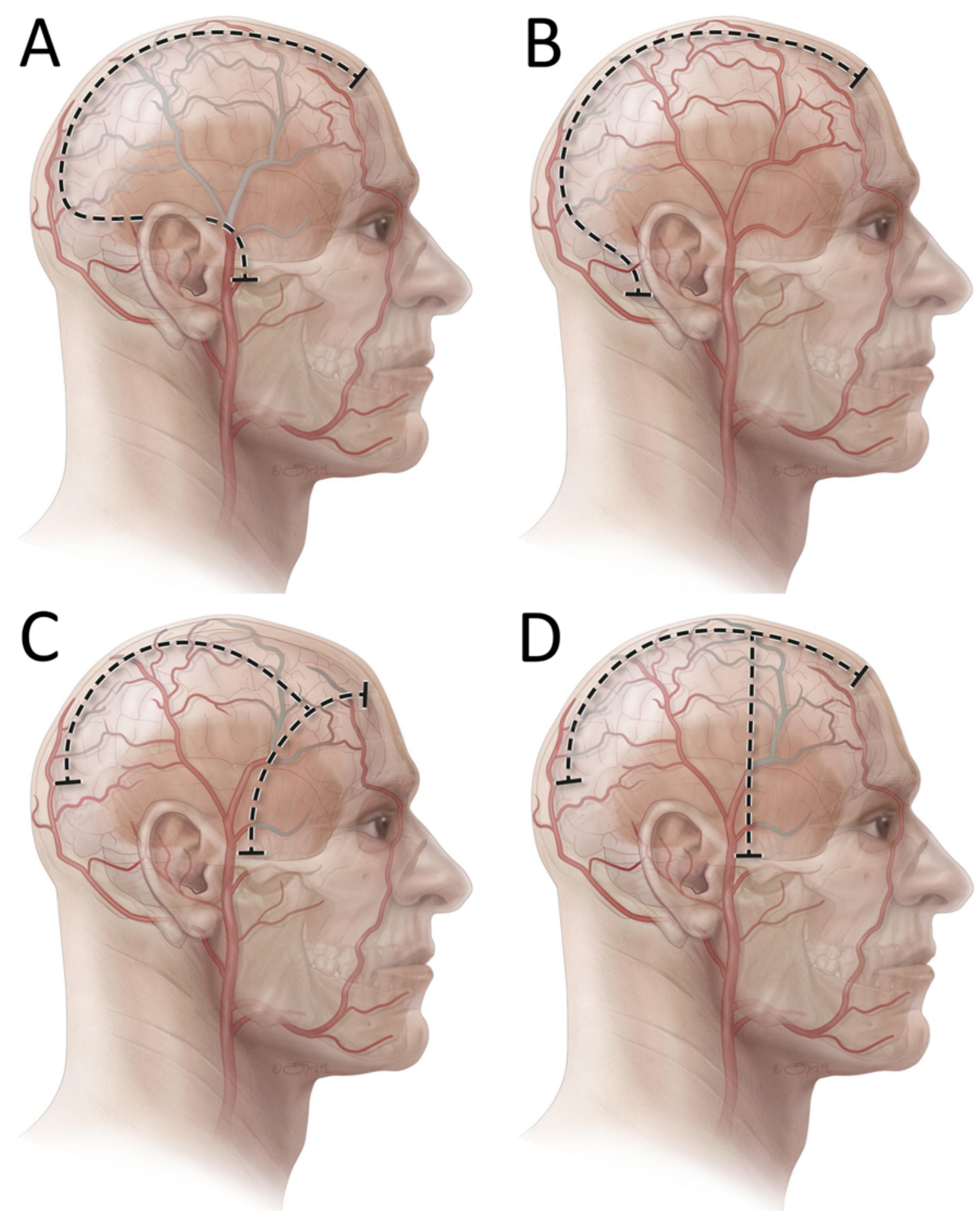

FIG. 1. Illustrations of incision types for DHC and subsequent CP. A: The classic trauma flap question-mark incision. B: The altered posterior question-mark incision. C: The "n-type" incision. D: The "T-type" or "Kempe" incision. Copyright Dirk Traufelder. Published with permission.

87) and altered posterior question-mark incision subgroup ( $\mathrm{n}=79$ ) were further compared. SSI was observed in 16 patients $(18.4 \%)$ in the question-mark subgroup versus 5 patients $(6.3 \%)$ in the posterior question-mark subgroup $(p=0.021)$. Consistent with results from previous trials, patient-specific risk factors such as obesity, DM, smoking, arterial disease, hypertension, and alcohol and drug abuse did not have any effect on SSI occurrence. The occurrence of an SSI after the initial DHC, the presence of an EVD during the first ICU stay, the length of the initial ICU stay, and the need for permanent shunting also had no effect on the post-CP SSI incidence. Slight elevation of the WBC count and CRP pre-CP did not increase the incidence of post-CP SSI. 


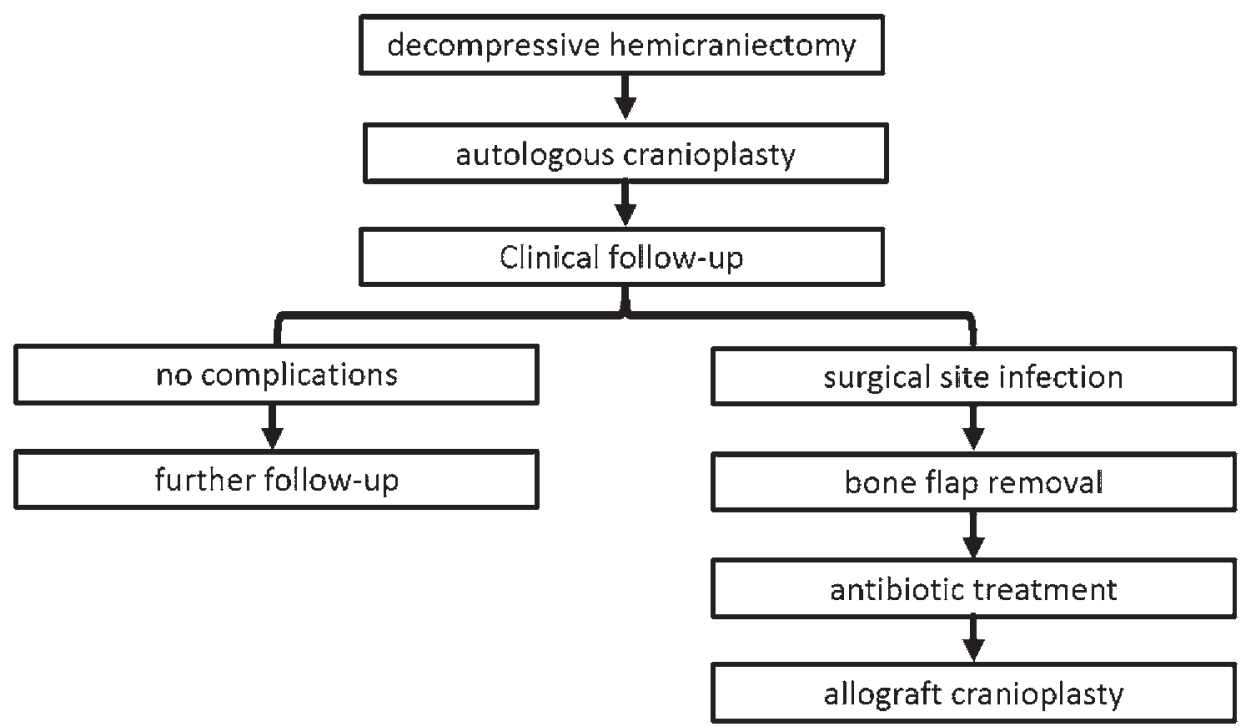

FIG. 2. Institutional treatment algorithm.

\section{Secondary Outcome}

In the multivariate analysis, the duration of $\mathrm{CP}$ surgery was significantly related to aBR $(p=0.032)$ and need for allograft $\mathrm{CP}(\mathrm{p}=0.019)$. Although the univariate analysis showed that surgical time for CP was longer in patients who developed bone resorption in the later course, the difference was not significant $(\mathrm{p}=0.078)$. Other surgeryspecific factors such as timing of $\mathrm{CP}$ or duration of DHC surgery, as well as patient-specific factors such as hydrocephalus, unfavorable outcome in the GOS-E, or confinement to bed had no effect on aBR occurrence. The only significant predictor of aBR was patient age, in which those developing resorption were on average 16 years younger $(\mathrm{p}<0.001)$ than those without aBR. It should be noted that the overall time of follow-up was significantly shorter in the posterior question-mark group compared to the question-mark group ( $809 \pm 468$ vs $1301 \pm 741$ days, $p$ $=0.001)$. The $\mathrm{CP}$ operative duration in patients with a posterior question-mark incision was on average 15 minutes longer. All results are depicted in Tables 1 and 2.

\section{Discussion}

In this observational analysis we have shown that the incision type is a significant predictor of post-CP SSI. The altered posterior question-mark incision, starting behind instead of in front of the ear, was shown to offer a protective effect against $\mathrm{CP}$ failure. We hypothesize that the

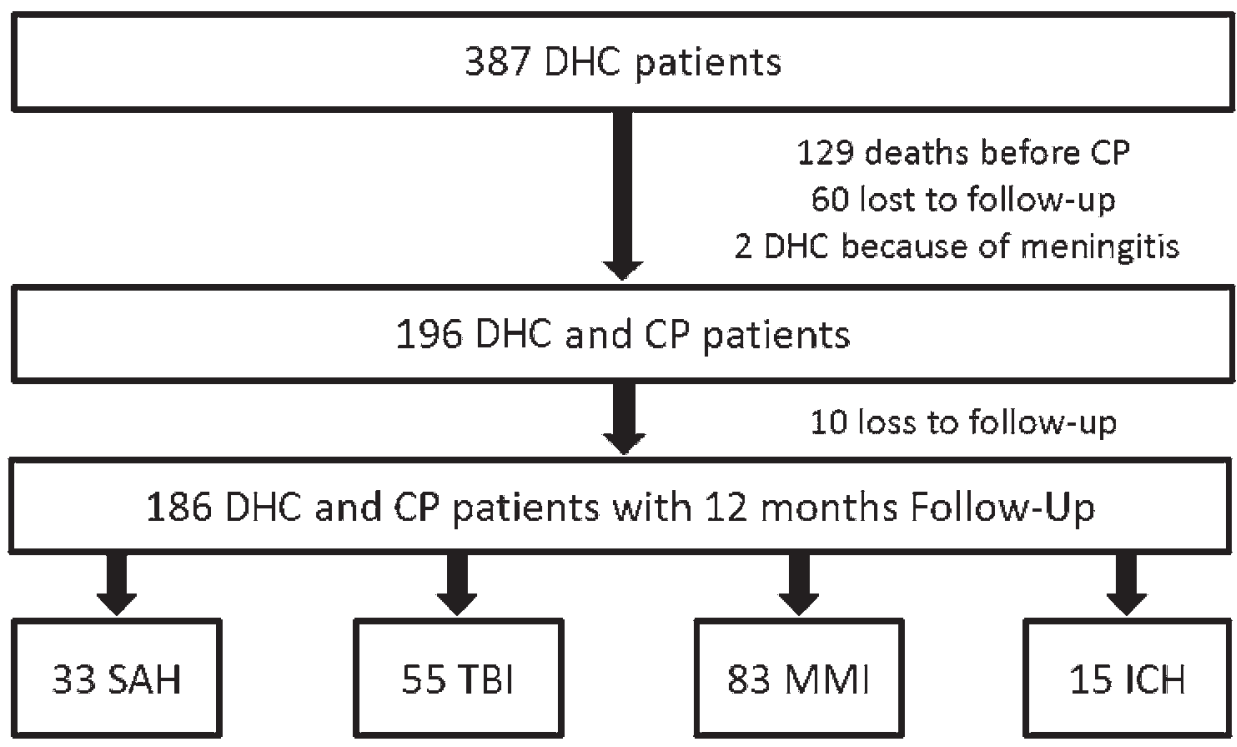

FIG. 3. Patient recruitment flow chart. 
TABLE 1. Baseline characteristics, patient- and surgery-specific factors

\begin{tabular}{|c|c|c|c|c|}
\hline Variable & All $(n=186)$ & No SSI $(n=161)$ & SSI $(n=25)$ & $p$ Value* \\
\hline Mean age $\pm S D$, yrs & $49 \pm 16$ & $50 \pm 16$ & $46 \pm 15$ & 0.226 \\
\hline Sex, $n(\%)$ & & & & 0.879 \\
\hline Females & $77(41.4)$ & $67(41.6)$ & $10(40)$ & \\
\hline Males & $109(58.6)$ & $94(58.4)$ & $15(60)$ & \\
\hline Indication for $\mathrm{DHC}, \mathrm{n}(\%)$ & & & & 0.986 \\
\hline $\mathrm{MMI}$ & $83(44.6)$ & $71(44.1)$ & $12(48.0)$ & \\
\hline $\mathrm{TBI}$ & $55(29.6)$ & $48(29.8)$ & $7(28.0)$ & \\
\hline SAH & $33(17.7)$ & $29(18.0)$ & $4(16.0)$ & \\
\hline $\mathrm{ICH}$ & $15(8.1)$ & $13(8.1)$ & $2(8.0)$ & \\
\hline \multicolumn{5}{|l|}{ Patient-specific factors, $\mathrm{n}(\%)$} \\
\hline $\mathrm{BMI}>30 \mathrm{~kg} / \mathrm{m}^{2}$ & $26(14.0)$ & $23(14.3)$ & $3(12.0)$ & 0.759 \\
\hline DM II & $14(7.5)$ & $13(8.1)$ & $1(4.0)$ & 0.472 \\
\hline Smoking & $59(31.7)$ & $55(34.2)$ & $4(16.0)$ & 0.069 \\
\hline CAD/PAD & $27(14.5)$ & $20(12.4)$ & $2(8.0)$ & 0.524 \\
\hline Hypertension & $94(50.5)$ & $85(52.8)$ & $9(36.0)$ & 0.118 \\
\hline Alcohol abuse & $19(10.2)$ & $17(10.6)$ & $2(8.0)$ & 0.694 \\
\hline Illicit drug use & $12(6.5)$ & $10(6.2)$ & $2(8.0)$ & 0.735 \\
\hline \multicolumn{5}{|l|}{ Surgery-specific factors, n (\%) } \\
\hline Mean duration of $\mathrm{DHC} \pm \mathrm{SD}$, mins & $104 \pm 63$ & $106.5 \pm 68.5$ & $93.5 \pm 36.8$ & 0.344 \\
\hline Post-DHC SSI & $17(9.1)$ & $14(8.7)$ & $4(16.0)$ & 0.250 \\
\hline Post-DHC hygroma & $78(41.9)$ & $70(43.5)$ & $8(32.0)$ & 0.279 \\
\hline Post-DHC infections overall & $123(66.1)$ & $109(67.7)$ & $14(56.0)$ & 0.488 \\
\hline UTI & $34(18.3)$ & & & \\
\hline Pneumonia & $98(52.7)$ & & & \\
\hline Sepsis & $14(7.5)$ & & & \\
\hline Other & $5(2.7)$ & & & \\
\hline EVD & $59(31.7)$ & $51(31.7)$ & $6(24.0)$ & 0.439 \\
\hline Mean ICU length of stay \pm SD, days & $27 \pm 19$ & $26.8 \pm 19.1$ & $29.4 \pm 21.0$ & 0.477 \\
\hline Early CP & $61(32.8)$ & $51(31.7)$ & $10(40.0)$ & 0.410 \\
\hline Permanent shunt & $48(25.8)$ & $47(29.2)$ & $8(32.0)$ & 0.775 \\
\hline Pre-CP leukocytosis (>10.0/nl) & $25(13.4)$ & $23(14.3)$ & $2(8.0)$ & 0.380 \\
\hline Pre-CP > CRP (>5 mg/L) & $57(30.6)$ & $51(31.7)$ & $6(24.0)$ & 0.439 \\
\hline Unfavorable GOS-E score at CP, 1-4 & $146(78.5)$ & $128(79.5)$ & $18(72.0)$ & 0.396 \\
\hline Bedridden at $\mathrm{CP}$ & $106(57.0)$ & $93(57.8)$ & $13(52.0)$ & 0.588 \\
\hline Mean duration of $\mathrm{CP} \pm \mathrm{SD}$, mins & $144 \pm 46$ & $146 \pm 45$ & $139 \pm 52$ & 0.471 \\
\hline Mean duration of follow-up $\pm S D$, days & $1153 \pm 703$ & $1159 \pm 700$ & $1125 \pm 736$ & 0.848 \\
\hline
\end{tabular}

$\mathrm{CAD}=$ coronary artery disease; $\mathrm{DM} I \mathrm{II}=$ diabetes mellitus type $2 ; \mathrm{PAD}=$ peripheral artery disease; $\mathrm{UTI}=$ urinary tract infection.

* Comparison between the no-SSI and SSI groups.

better scalp vascularization due to complete preservation of the STA and partial preservation of the occipital artery is the main contributing factor. Thus, we were able to identify an easily applicable technique to reduce the CP infection risk. Based on these results, our department has abandoned the classic inverted question-mark incision in favor of the altered posterior type for DHC. A minor drawback of this altered incision type is a limited exposure of the anterior temporal region, due to the fact that the skin flap, when being folded over and retracted, is hindered by the attachment at the level of the external auditory canal. How pronounced this problem is varies considerably depending on general skull morphology. However, as the temporal squama is removed with a rongeur in both the classic as well as the posterior incision type, this minor limitation does not compromise the ability to fulfill an adequate temporal decompression.

It should be noted that no significant difference in SSI rates after DHC were observed between the classic and posterior incision types. This could be attributed to the fact that the rate of wound infection after the initial decompression is low, making intergroup differences less explicit.

Some authors advocate the n-type incision as a first 
TABLE 2. Results comparing the question-mark and posterior question-mark incision subgroups

\begin{tabular}{|c|c|c|c|c|}
\hline Variable & T- or n-Type $(n=20)$ & Question Mark $(\mathrm{n}=87$ ) & Posterior Question Mark $(n=79)$ & p Value* \\
\hline Mean age \pm SD (range), yrs & $49 \pm 15$ & $52 \pm 16$ & $47 \pm 16$ & 0.092 \\
\hline Sex, n (\%) & & & & 0.420 \\
\hline Females & $4(20)$ & $29(33.3)$ & $32(40.5)$ & \\
\hline Males & $16(80)$ & $58(66.7)$ & $47(59.5)$ & \\
\hline Indication for $\mathrm{DHC}, \mathrm{n}(\%)$ & & & & 0.192 \\
\hline $\mathrm{MMI}$ & $0(0.0)$ & $46(52.9)$ & $37(46.8)$ & \\
\hline $\mathrm{TBI}$ & $0(0.0)$ & $31(35.6)$ & $24(30.4)$ & \\
\hline $\mathrm{SAH}$ & $17(85)$ & $7(8.0)$ & $9(11.4)$ & \\
\hline $\mathrm{ICH}$ & $3(15)$ & $3(3.4)$ & $9(11.4)$ & \\
\hline \multicolumn{5}{|l|}{ Surgery-specific risk factors, $\mathrm{n}(\%)$} \\
\hline Mean duration of $\mathrm{DHC} \pm \mathrm{SD}$, mins & $106 \pm 47$ & $105 \pm 79$ & $102 \pm 47$ & 0.843 \\
\hline Post-DHC SSI & $2(10.0)$ & $9(10.3)$ & $6(7.6)$ & 0.597 \\
\hline Post-DHC hygroma & $11(55.0)$ & $35(40.2)$ & $32(40.5)$ & 0.972 \\
\hline Post-DHC infections overall & $17(85.0)$ & $52(59.8)$ & $54(68.3)$ & 0.415 \\
\hline UTI & $3(15)$ & $14(16.1)$ & $17(21.5)$ & \\
\hline Pneumonia & $13(65)$ & $43(49.4)$ & $42(53.2)$ & \\
\hline Sepsis & $2(10.0)$ & $3(3.4)$ & $9(11.4)$ & \\
\hline Other & $1(5.0)$ & $2(2.3)$ & $1(1.3)$ & \\
\hline EVD & $18(90.0)$ & $20(23.0)$ & $21(26.6)$ & 0.476 \\
\hline Mean ICU length of stay \pm SD, days & $32 \pm 15$ & $22 \pm 18$ & $30 \pm 21$ & 0.010 \\
\hline Early $\mathrm{CP}$ & $9(45.0)$ & $31(35.6)$ & $21(26.6)$ & 0.243 \\
\hline Permanent shunt & $9(45.0)$ & $18(20.7)$ & $21(26.6)$ & 0.604 \\
\hline Pre-CP leukocytosis (>10.0/nl) & $2(10.0)$ & $9(10.3)$ & $14(17.7)$ & 0.261 \\
\hline Pre-CP > CRP (>5 mg/L) & $4(20.0)$ & $20(23.0)$ & $33(41.8)$ & 0.012 \\
\hline Unfavorable GOS-E at CP, 1-4 & $14(70.0)$ & $60(69.0)$ & $72(91.1)$ & 0.001 \\
\hline Bedridden at $\mathrm{CP}$ & $11(55)$ & $46(52.9)$ & $49(62.0)$ & 0.273 \\
\hline Mean duration of $\mathrm{CP} \pm \mathrm{SD}$, mins & $174 \pm 38$ & $134 \pm 47$ & $149 \pm 43$ & 0.023 \\
\hline Mean duration of follow-up $\pm S D$, days & $1011 \pm 900$ & $1301 \pm 741$ & $809 \pm 468$ & 0.001 \\
\hline Post-CP SSI & $4(20.0)$ & $16(18.4)$ & $5(6.3)$ & 0.021 \\
\hline Post-CP aBR & $5(25.0)$ & $15(17.2)$ & $12(15.2)$ & 0.834 \\
\hline Post-CP aBR triggering treatment & $2(10.0)$ & $4(4.6)$ & $6(7.6)$ & 0.525 \\
\hline Allograft CP & $4(20)$ & $25(28.7)$ & $14(17.7)$ & 0.126 \\
\hline
\end{tabular}

Boldface type indicates statistical significance.

* Comparison between the question-mark and posterior question-mark incision groups.

choice. ${ }^{28}$ In our series, the infection rate of this incision was comparable to that of the classic question-mark incision and should therefore only be reserved for cases in which a previous curvilinear frontotemporal incision forces one to do so. In this cohort, however, the n-type incision was never used as a first option, so results could be different if used as the primary incision type.

The only significant predictor of aBR was patient age. Revision surgery with allograft CP was necessary in 13 cases $(7.0 \%)$; of those, 8 cases were younger than 30 years. Conversely, there were 20 patients younger than 30 in the cohort, of whom the other 12 did not show relevant bone resorption. In this case, the subjectivity in defining what is relevant resorption is relevant and creates observer and detection bias. Some authors advocate the primary use of allograft CP in younger patients. ${ }^{32}$ A cost-effectiveness analysis could help shed light on this dilemma. Due to the still high success rate, low cost, and availability of this procedure, we still opt for primary autologous $\mathrm{CP}$, also in younger patients.

Although $C$.acnes has been identified as a cause of osteolysis, possibly due to a slow low-grade infection, it was treated in our series as a contaminant. This was justified by the lack of additional inflammatory signs. The results of the recent analysis by Butenschoen et al. ${ }^{34}$ should, however, trigger more vigilance toward less virulent bacteria in patients with $\mathrm{CP}$.

We anticipated that unfavorable GOS-E scores and especially confinement to bed would have a promoting effect on wound healing problems and infection. This belief comes from the observation that in many cases, wound dehiscence or infection sites in bedridden patients appear in 
the occipital region where continuous pressure decreases blood flow. In our analysis, this hypothesis was not confirmed. However, vigilant attention should continue to be paid to patients who are not able to indicate signs of postoperative complications and discomfort themselves.

Obvious weaknesses of this study are its observational and retrospective nature without randomization. Even if subgroups proved to be comparable, due to the long recruitment period and heterogeneous follow-up, there is the possibility of selection as well as detection bias. The minimal duration of follow-up was arbitrarily set at 12 months. It could be that late SSI and aBR in the posterior questionmark group was not reported due to the shorter follow-up. Nonetheless, the average follow-up of the posterior question-mark group still exceeds 2 years, and due to the low prevalence of late SSI, the bias caused by this difference in follow-up is estimated to be minimal.

In general, the follow-up duration might have been too short to identify all cases of aBR, as a previous study showed that bone flap resorption occurred in a pediatric population after an average of 13.3 months after primary CP. ${ }^{33}$ Additionally, being a single-center study, these results cannot simply be extrapolated to other institutions because of potentially other unidentifiable factors involved. Finally, some relevant factors have not been examined, such as cosmetic result, nonspecific operative complications such as deep vein thrombosis, or the seniority of the operating surgeon. However, in an observational trial, supervised residents were as likely as board-certified neurosurgeons to have postoperative complications. ${ }^{10}$

\section{Conclusions}

Complications after autologous $\mathrm{CP}$ for patients who were treated by DHC are common and bothersome. This trial focuses on CP complications exclusively after DHC. We showed that the altered posterior question-mark incision is associated with a lower infection and $\mathrm{CP}$ failure rate. Based on these results, the classic question-mark incision in our institution has been superseded by the altered posterior type.

\section{References}

1. Hutchinson PJ, Kolias AG, Tajsic T, et al. Consensus statement from the International Consensus Meeting on the Role of Decompressive Craniectomy in the Management of Traumatic Brain Injury: consensus statement. Acta Neurochir (Wien). 2019;161(7):1261-1274.

2. Hofmeijer J, Kappelle LJ, Algra A, et al. Surgical decompression for space-occupying cerebral infarction (the Hemicraniectomy After Middle Cerebral Artery infarction with Lifethreatening Edema Trial [HAMLET]): a multicentre, open, randomised trial. Lancet Neurol. 2009;8(4):326-333.

3. Jüttler E, Schwab S, Schmiedek P, et al. Decompressive Surgery for the Treatment of Malignant Infarction of the Middle Cerebral Artery (DESTINY): a randomized, controlled trial. Stroke. 2007;38(9):2518-2525.

4. Vahedi K, Vicaut E, Mateo J, et al. Sequential-design, multicenter, randomized, controlled trial of early decompressive craniectomy in malignant middle cerebral artery infarction (DECIMAL Trial). Stroke. 2007;38(9):2506-2517.

5. Goedemans T, Verbaan D, Coert BA, et al. Decompressive craniectomy in aneurysmal subarachnoid haemorrhage for hematoma or oedema versus secondary infarction. $\mathrm{Br} \mathrm{J} \mathrm{Neu-}$ rosurg. 2018;32(2):149-156.

6. Güresir E, Raabe A, Setzer M, et al. Decompressive hemicraniectomy in subarachnoid haemorrhage: the influence of infarction, haemorrhage and brain swelling. J Neurol Neurosurg Psychiatry. 2009;80(7):799-801.

7. Fung C, Murek M, Z'Graggen WJ, et al. Decompressive hemicraniectomy in patients with supratentorial intracerebral hemorrhage. Stroke. 2012;43(12):3207-3211.

8. Takeuchi S, Wada K, Nagatani K, et al. Decompressive hemicraniectomy for spontaneous intracerebral hemorrhage. $\mathrm{Neu}$ rosurg Focus. 2013;34(5):E5.

9. Malcolm JG, Rindler RS, Chu JK, et al. Early cranioplasty is associated with greater neurological improvement: a systematic review and meta-analysis. Neurosurgery. 2018;82(3):278288.

10. Honeybul S, Ho KM. Cranioplasty: morbidity and failure. $\mathrm{Br}$ J Neurosurg. 2016;30(5):523-528.

11. Herteleer M, Ectors N, Duflou J, Van Calenbergh F. Complications of skull reconstruction after decompressive craniectomy. Acta Chir Belg. 2017;117(3):149-156.

12. Klinger DR, Madden C, Beshay J, et al. Autologous and acrylic cranioplasty: a review of 10 years and 258 cases. World Neurosurg. 2014;82(3-4):e525-e530.

13. Shibahashi K, Hoda H, Takasu Y, et al. Cranioplasty outcomes and analysis of the factors influencing surgical site infection: a retrospective review of more than 10 years of institutional experience. World Neurosurg. 2017;101:20-25.

14. Zanaty M, Chalouhi N, Starke RM, et al. Complications following cranioplasty: incidence and predictors in 348 cases. $J$ Neurosurg. 2015;123(1):182-188.

15. Kim JS, Park IS, Kim SK, et al. Analysis of the risk factors affecting the surgical site infection after cranioplasty following decompressive craniectomy. Korean J Neurotrauma. 2015;11(2):100-105.

16. Kim SH, Kang DS, Cheong JH, et al. Comparison of complications following cranioplasty using a sterilized autologous bone flap or polymethyl methacrylate. Korean J Neurotrauma. 2017;13(1):15-23.

17. Mukherjee S, Thakur B, Haq I, et al. Complications of titanium cranioplasty - a retrospective analysis of 174 patients. Acta Neurochir(Wien). 2014;156(5):989-998.

18. Rosenthal G, Ng I, Moscovici S, et al. Polyetheretherketone implants for the repair of large cranial defects: a 3-center experience. Neurosurgery. 2014;75(5):523-529.

19. Jin SW, Kim SD, Ha SK, et al. Analysis of the factors affecting surgical site infection and bone flap resorption after cranioplasty with autologous cryopreserved bone: the importance of temporalis muscle preservation. Turk Neurosurg. 2018;28(6):882-888.

20. Kim H, Sung SO, Kim SJ, et al. Analysis of the factors affecting graft infection after cranioplasty. Acta Neurochir (Wien). 2013;155(11):2171-2176.

21. Sundseth J, Sundseth A, Berg-Johnsen J, et al. Cranioplasty with autologous cryopreserved bone after decompressive craniectomy: complications and risk factors for developing surgical site infection. Acta Neurochir (Wien). 2014;156(4):805811.

22. Abode-Iyamah KO, Chiang HY, Winslow N, et al. Risk factors for surgical site infections and assessment of vancomycin powder as a preventive measure in patients undergoing firsttime cranioplasty. J Neurosurg. 2018;128(4):1241-1249.

23. Güresir E, Vatter H, Schuss P, et al. Rapid closure technique in decompressive craniectomy. J Neurosurg. 2011;114(4):954-960.

24. Vieira E, Guimarães TC, Faquini IV, et al. Randomized controlled study comparing 2 surgical techniques for decompressive craniectomy: with watertight duraplasty and without watertight duraplasty. J Neurosurg. 2018;129(4):1017-1023. 
25. Tanrikulu L, Oez-Tanrikulu A, Weiss C, et al. The bigger, the better? About the size of decompressive hemicraniectomies. Clin Neurol Neurosurg. 2015;135:15-21.

26. Malcolm JG, Rindler RS, Chu JK, et al. Complications following cranioplasty and relationship to timing: a systematic review and meta-analysis. J Clin Neurosci. 2016;33:39-51.

27. Lyon KA, Patel NP, Zhang Y, et al. Novel hemicraniectomy technique for malignant middle cerebral artery infarction: technical note. Oper Neurosurg (Hagerstown). 2019;17(3):273-276.

28. Yang HS, Hyun D, Oh CH, et al. A faster and wider skin incision technique for decompressive craniectomy: n-shaped incision for decompressive craniectomy. Korean J Neurotrauma. 2016;12(2):72-76.

29. Mustroph CM, Malcolm JG, Rindler RS, et al. Cranioplasty infection and resorption are associated with the presence of a ventriculoperitoneal shunt: a systematic review and metaanalysis. World Neurosurg. 2017;103:686-693.

30. Schuss P, Borger V, Güresir Á, et al. Cranioplasty and ventriculoperitoneal shunt placement after decompressive craniectomy: staged surgery is associated with fewer postoperative complications. World Neurosurg. 2015;84(4):1051-1054.

31. Yang XF, Wang H, Wen L, et al. The safety of simultaneous cranioplasty and shunt implantation. Brain Inj. 2017;31(12):1651-1655.

32. Schwarz F, Dünisch P, Walter J, et al. Cranioplasty after decompressive craniectomy: is there a rationale for an initial artificial bone-substitute implant? A single-center experience after 631 procedures. J Neurosurg. 2016;124(3):710-715.

33. Grant GA, Jolley M, Ellenbogen RG, et al. Failure of autologous bone-assisted cranioplasty following decompressive craniectomy in children and adolescents. J Neurosurg. 2004;100(2)(Suppl Pediatrics):163-168.
34. Butenschoen VM, Seifert M, Meyer B, et al. Presence of Propionibacterium acnes in patients with aseptic bone graft resorption after cranioplasty: preliminary evidence for lowgrade infection. J Neurosurg. Published online August 30, 2019. doi:10.3171/2019.5.JNS191200

\section{Disclosures}

The authors report no conflict of interest concerning the materials or methods used in this study or the findings specified in this paper.

\section{Author Contributions}

Conception and design: Veldeman, Clusmann. Acquisition of data: Veldeman, Daleiden, Hamou. Analysis and interpretation of data: Veldeman, Daleiden, Hamou, Clusmann. Drafting the article: Veldeman, Daleiden. Critically revising the article: Veldeman, Daleiden, Hamou, Clusmann. Reviewed submitted version of manuscript: Veldeman, Daleiden, Hoellig, Clusmann. Approved the final version of the manuscript on behalf of all authors: Veldeman. Statistical analysis: Veldeman, Hoellig. Administrative/technical/material support: Veldeman, Hoellig, Clusmann. Study supervision: Veldeman, Hoellig, Clusmann.

\section{Correspondence}

Michael Veldeman: RWTH Aachen University Hospital, Aachen, Germany.mveldeman@ukaachen.de. 